\title{
Oxidación de antimonio del mineral de pirargirita con sulfuro de sodio
}

\author{
Y. P. Gómez Espinoza ${ }^{1}$, M. Reyes Pérezi*, A. M. Teja Ruiz², M. Pérez Labra ${ }^{1}$ \\ F. R. Barrientos Hernández ${ }^{1}$, J. C. Juárez Tapia ${ }^{1}$, V. E. Reyes Cruz ${ }^{1}$
}

\begin{abstract}
1Área Académica de Ciencias de la Tierra y Materiales, Universidad Autónoma del Estado de Hidalgo, Mineral de la Reforma, Hidalgo, 42184, México. yessicagmz13@gmail.com, miguelabra@hotmail.com, frbh68@hotmail.com, jutj731101@hotmail.com, reyescruz16@yahoo.com

${ }^{2}$ Escuela Superior de Ingeniería Química e Industrias Extractivas ESIQUIE, Instituto Politécnico Nacional, Unidad profesional, Adolfo López Mateos, 07738, México. ice9791@gmail.com
\end{abstract}

*Autor de correspondencia: mreyes@uaeh.edu.mx

\section{RESUMEN}

La pirargirita es una sulfosal de plata de formula $\mathrm{Ag}_{3} \mathrm{SbS}_{3}$ caracterizada por su alto contenido de plata, lo cual la convierte en una importante fuente de este metal, sin embargo, esta fase se considera altamente refractaria a la lixiviación de plata con cianuro. Por tal motivo se estudió la oxidación de pirargirita bajo diferentes concentraciones de sulfuro de sodio nona hidratado, $\mathrm{Na}_{2} \mathrm{~S} .9 \mathrm{H}_{2} \mathrm{O}$ y tiempos de reacción, los sólidos resultantes de todas las pruebas se caracterizaron por técnicas de: microscopia, difracción de rayos $X$ y espectroscopia de infrarrojo por transformada de Fourier. El análisis por MEB- EDS y DRX confirmaron la presencia de una única fase cristalina de pirargirita con la siguiente composición 58.54 \% de Ag, 17.01 de S, 19.49 de $\mathrm{Sb}$ y $\mathrm{Cu} 4.95 \%$. Los sólidos obtenidos después de cada prueba de oxidación del antimonio con sulfuro de sodio, se caracterizaron y muestran claramente la presencia de sulfuro de plata $\mathrm{Ag}_{2} \mathrm{~S}$, las fases oxidadas de antimonio no se detectaron por esta vía, sin embargo, la técnica FTIR pudo verificar la formación de enlaces $\mathrm{Sb}-\mathrm{O}$ del $\mathrm{Sb}_{2} \mathrm{O}_{3}$ con las principales bandas de absorción en: 1384, 1019, 534, $760 \mathrm{~cm}^{-1}$.

Palabras Clave: Oxidación, Antimonio, Sulfuro de Sodio, Pirargirita, Sulfuro de Plata.

\section{INTRODUCCIÓN}

La plata, se asocia frecuentemente con sulfuros polimetálicos y sulfosales, la fase sulfurosa más conocida es la acantita $\mathrm{Ag}_{2} \mathrm{~S}$ sulfuro de plata, otros minerales que contienen plata son la pirargirita, $\mathrm{Ag}_{3} \mathrm{SbS}_{3}$, proustita $\mathrm{Ag}_{3} \mathrm{AsS}_{3}$, aguilarita $\mathrm{Ag}_{4} \mathrm{SeS}$, tenantita $\mathrm{Cu}_{6}\left(\mathrm{Cu}_{4}(\mathrm{Fe}, \mathrm{Zn})_{2}\right) \mathrm{As}_{4} \mathrm{~S}_{13}$ y tetraedrita (Cu, Fe, $\left.\mathrm{Ag}, \mathrm{Zn}\right)_{12} \mathrm{Sb}_{14} \mathrm{~S}_{13}$ con inclusiones de plata [1]. El mineral más común que contiene plata es la acantita y responde fácilmente a la lixiviación con cianuro [2].

Sin embargo, se reporta en la literatura, la refractariedad que presentan algunos minerales que contienen plata y antimonio, esto debido al efecto retardante causado por los enlaces $\mathrm{Ag}-\mathrm{Sb}$ y $\mathrm{Ag}_{2} \mathrm{~S}$ [3]. Se entiende como mineral refractario aquel que tiene una escaza disolución de la plata del mineral. Estas dificultades por efecto de la refractariedad que presentan los minerales como por ejemplo la pirargirita, se ha tratado de superar mediante tratamientos pirometalurgicos como la tostación [4]. 
Así como la ruta hidrometalúrgica, que constituye la oxidación a presión y lixiviación con bacterias. No obstante, la primera vía involucra la generación de gases de reacción como $\mathrm{SO}_{2}$ o óxidos de antimonio volátiles, mientras que la vía acuosa contiene dificultades, como elevados costos de operación y largos periodos de reacción [4].

La pirargirita es un mineral rico en plata con contenidos de alrededor del $59 \%$, por lo tanto, debido a su característica de refractariedad, se han promovido rutas alternas de tratamiento con variantes de las anteriormente descritas[5], tal como lo es un tratamiento previo con un agente oxidante como lo es el ozono antes de la lixiviación con cianuro. Los resultados encontrados indican que tanto la plata como el antimonio se disuelven en porcentaje considerable del $80 \%$, bajo condiciones de $0.18 \mathrm{M}$ de ácido sulfúrico y $0.079 \mathrm{~g}$ de ozono $\left(\mathrm{O}_{3}\right)$ por litro [4].

Las fuertes condiciones ácidas del proceso mencionado, podrían generar repercusiones tanto de la índole económica como medio ambientales. En otro trabajo se investigó el proceso de pre tratamiento de la molienda intensiva, buscando la activación mecánica de los componentes del mineral refractario. Esta, produce cambios significativos en la superficie del mineral que influyen decisivamente en la refractariedad, sin embargo, los tiempos prolongados de molienda son un inconveniente para este proceso [6].

La integración del proceso anterior, consiste en una lixiviación mecano química, la cual involucra operaciones de molienda y lixiviación en una sola etapa, lo cual mejoraría el desarrollo de la molienda activa [6]. En otro proceso, se ha estudiado la lixiviación de plata y antimonio de minerales como la tetraedrita $\mathrm{Cu}_{3} \mathrm{SbS}_{3}$ conteniendo plata, empleando sulfuro de sodio $\mathrm{Na}_{2} \mathrm{~S}$ e hidróxido de sodio $\mathrm{NaOH}$, describiendo la química de la lixiviación del mineral con $\mathrm{Na}_{2} \mathrm{~S}$ ecuación 1 [7].

$$
2 \mathrm{Cu}_{3} \mathrm{SbS}_{3}+\mathrm{Na}_{2} \mathrm{~S}=3 \mathrm{Cu}_{2} \mathrm{~S}+2 \mathrm{NaSbS}_{2}
$$

Por lo cual, en este trabajo de investigación, se estudia la oxidación de antimonio de un mineral de pirargirita, mediante el uso de diferentes concentraciones de sulfuro de sodio, caracterizando los productos formados después de la oxidación, por técnicas de difracción de rayos $X$ (DRX) y espectroscopía de infrarrojo por transformada de Fourier (FTIR).

\section{PARTE EXPERIMENTAL}

Para llevar a cabo esta investigación se empleó un mineral de pirargirita prácticamente puro, el cual se caracterizó previamente mediante microscopia electrónica de barrido (MEB) en conjunto con microanálisis por dispersión de energias (EDS), espectroscopía de Infrarrojo por transformada de Fourier (FTIR) y difracción de rayos X (DRX). El Mineral de pirargirita se pulverizó en un mortero de ágata para evitar contaminación del mineral, se tamizó vía húmeda empleando una serie de tamices Tyler, el tamaño de malla en particular utilizado para este trabajo fue la malla $400(37 \mu \mathrm{m})$.

Para todos los experimentos se usó $1 \mathrm{~g}$ de mineral de pirargirita, un reactor de vidrio y 1 $L$ de agua desionizada utilizando diferentes concentraciones de sulfuro de sodio, $0.416 \times 10^{-2}, 0.833 \times 10^{-2}, 1.25 \times 10^{-2}, 1.67 \times 10^{-2}, 2.08 \times 10^{-2}, 2.5 \times 10^{-2}$ y $2.91 \times 10^{-2} \mathrm{M}$, la 
pulpa mineral se agitó continuamente usando una propela de agitación de cuatro aspas inerte a la solución de lixiviación.

El tiempo de reacción fue de 12 horas, el sólido se dejó sedimentar, se decantó y se lavó copiosamente con abundante agua desionizada, posteriormente el mineral se dejó secar a temperatura ambiente y se caracterizó por las técnicas instrumentales anteriormente descritas. La reacción química esperada es como se muestra en la ecuación 2.

$$
2 \mathrm{Ag}_{3} \mathrm{SbS}_{3}+\mathrm{Na} 2 \mathrm{~S}+\mathrm{H}_{2} \mathrm{O}+1 / 2 \mathrm{O}_{2}=3 \mathrm{Ag}_{2} \mathrm{~S}+\mathrm{Sb}_{2} \mathrm{~S}_{4}{ }^{2-}+2 \mathrm{NaOH}
$$

\section{RESULTADOS}

El mineral de pirargirita usado en esta investigación se analizó vía MEB en conjunto con EDS y mapeos elementales, los resultados encontrados muestran que se trata de una sulfosal de plata de alta pureza del tipo pirargirita con contenidos de $58.54 \%$ de Ag,19.49 \% de Sb, $17.01 \%$ de S y $4.95 \%$ de Cu. La morfología de las partículas es alargada, con bordes lisos y con fractura tipo concoidea.
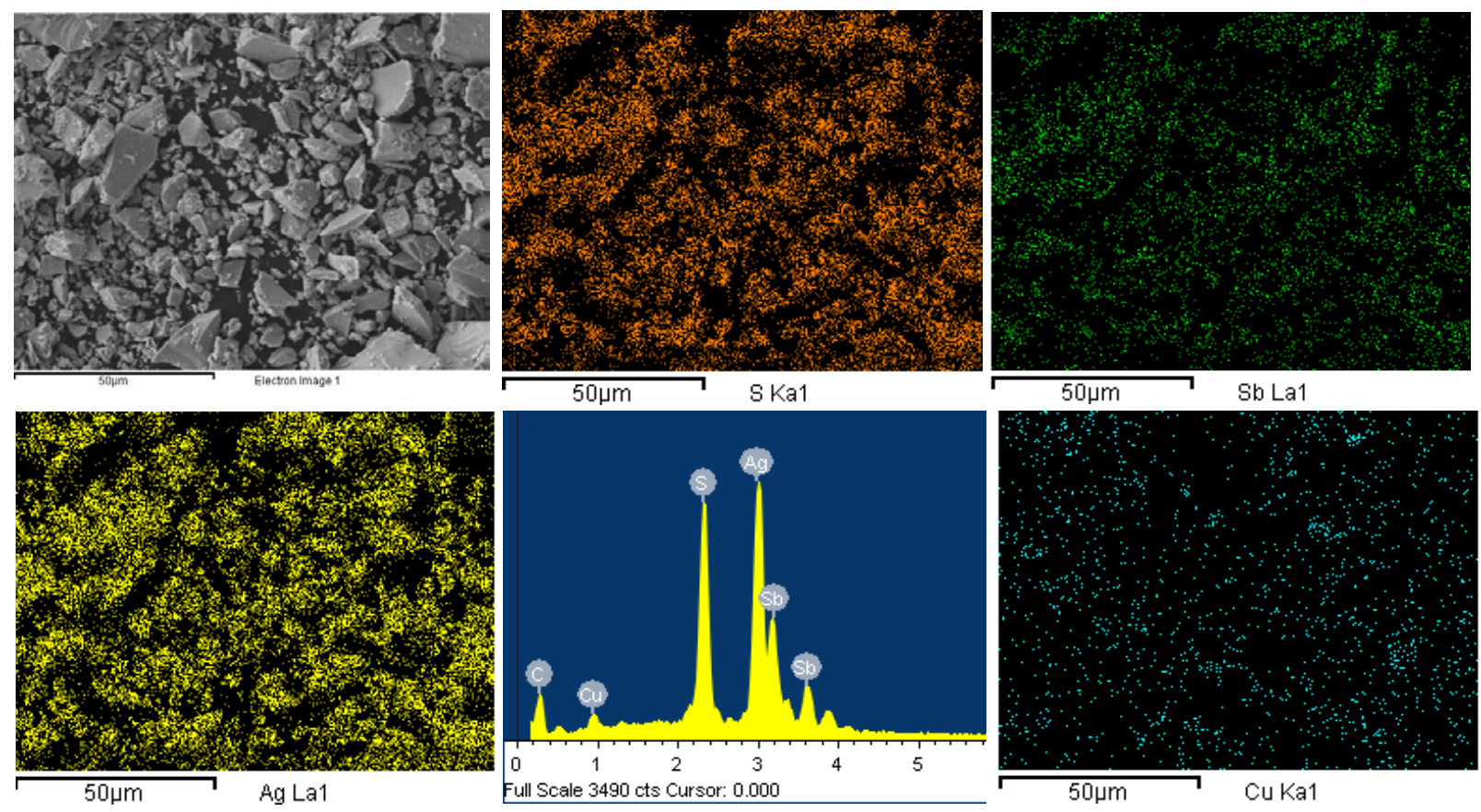

Figura 1. Micrografía de MEB, EDS y mapeos de los elementos mayoritarios del mineral.

La figura 2 muestra el espectro de difracción de rayos $X(D R X)$ del mineral de pirargirita con un tamaño de partícula de $37 \mu \mathrm{m}$ sin tratamiento indexado con el patrón de difracción PDF 00-019-1135 con el Software Match, el cual corresponde al de una pirargirita de alta pureza. Mientras, el espectro correspondiente al sólido resultante después de la oxidación de antimonio con sulfuro de sodio empleando una concentración de $2.5 \times 10^{-2} \mathrm{M}$ y tiempo de reacción de $12 \mathrm{~h}$, tal como se presenta en la ecuación 2 el $\mathrm{Na}_{2} \mathrm{~S} * 9 \mathrm{H}_{2} \mathrm{O}$ no solo lixivia al antimonio formando un sulfuro de antimonio 
acuoso estable a pH alcalino de 13, sino además, transforma la estructura del mineral de pirargirita de hexagonal a monoclínica correspondiente al sulfuro de plata PDF 96 $900-0254$.

Similares espectros se obtienen cuando la concentración del sulfuro de sodio es 2.91 $\times 10^{-2} \mathrm{M}$ y $12 \mathrm{~h}$ de reacción, menores concentraciones de sulfuro de sodio conducen a incrementar el tiempo de reacción y oxidar parcialmente el mineral de pirargirita. No obstante, la valiosa información obtenida en la técnica de difracción no aporta conclusiones acerca del estado del antimonio en el mineral, por tal razón los sólidos obtenidos se caracterizaron por FTIR.

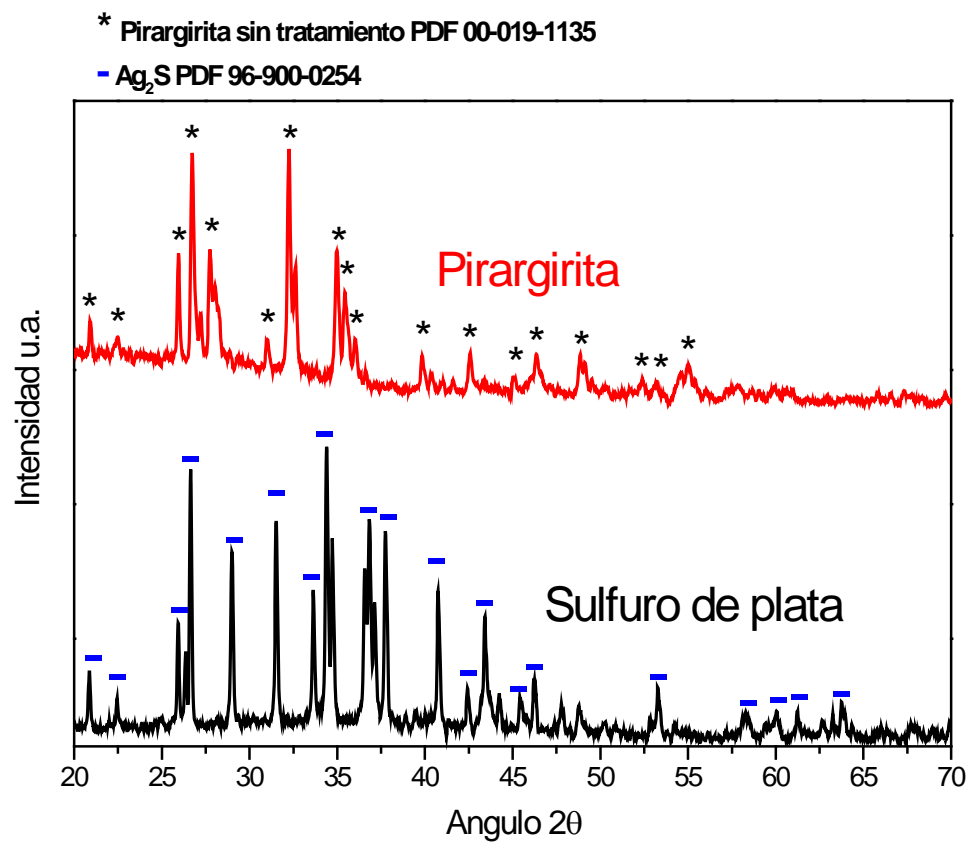

Figura 2. Espectros de DRX de la pirargirita sin oxidar y oxidada con $\mathrm{Na}_{2} \mathrm{~S}^{*} 9 \mathrm{H}_{2} \mathrm{O}, 2.5 \times 10^{-2} \mathrm{M}$

La figura 3 muestra los espectros de infrarrojo por transformada de Fourier FTIR de las partículas de pirargirita; sin tratamiento, oxidadas y el de un reactivo químico puro de $\mathrm{Sb}_{2} \mathrm{O}_{3}$. Cuando la pirargirita se pulveriza en el mortero las superficies frescas del mineral quedan expuestas a la atmosfera oxidante, formando especies como el ion sulfato enlazado de manera monodentada con el metal del mineral, esto es debido a la escisión de la banda principal de absorción del ion $\mathrm{SO}_{4}{ }^{-2}$ en tres bandas características en $1056 \mathrm{~cm}^{-1}, 1114 \mathrm{~cm}^{-1}$ y $1160 \mathrm{~cm}^{-1}$.

Además, la pirargirita solamente pulverizada, muestra la formación de débiles bandas de absorción en $534 \mathrm{~cm}^{-1}, 689 \mathrm{~cm}^{-1}, 1020 \mathrm{~cm}^{-1}$ y $1384 \mathrm{~cm}^{-1}$, correspondientes al enlace $\mathrm{Sb}-\mathrm{O}$ del trióxido de antimonio $\mathrm{Sb}_{2} \mathrm{O}_{3}$ la naturaleza de estos enlaces como primera aproximación están relacionados con la formación de una capa de pasivación, donde el óxido de antimonio III, crea una barrera que puede llegar a impedir el acceso del reactivo de lixiviación afectando el éxito de la cianuración. 
Con la oxidación del antimonio empleando sulfuro de sodio, se espera que en su totalidad se transforme en sulfuro de antimonio acuoso $\mathrm{Sb}_{2} \mathrm{~S}_{4}{ }^{2-}$ sin embargo, del espectro de infrarrojo de la pirargirita oxidada se observa que no es en su totalidad, demostrado por las intensas bandas de absorción de los enlaces $\mathrm{Sb}-\mathrm{O}$ del $\mathrm{Sb}_{2} \mathrm{O}_{3}$ en alrededor de 1384, 1020, 760, 689 y $534 \mathrm{~cm}^{-1}$.

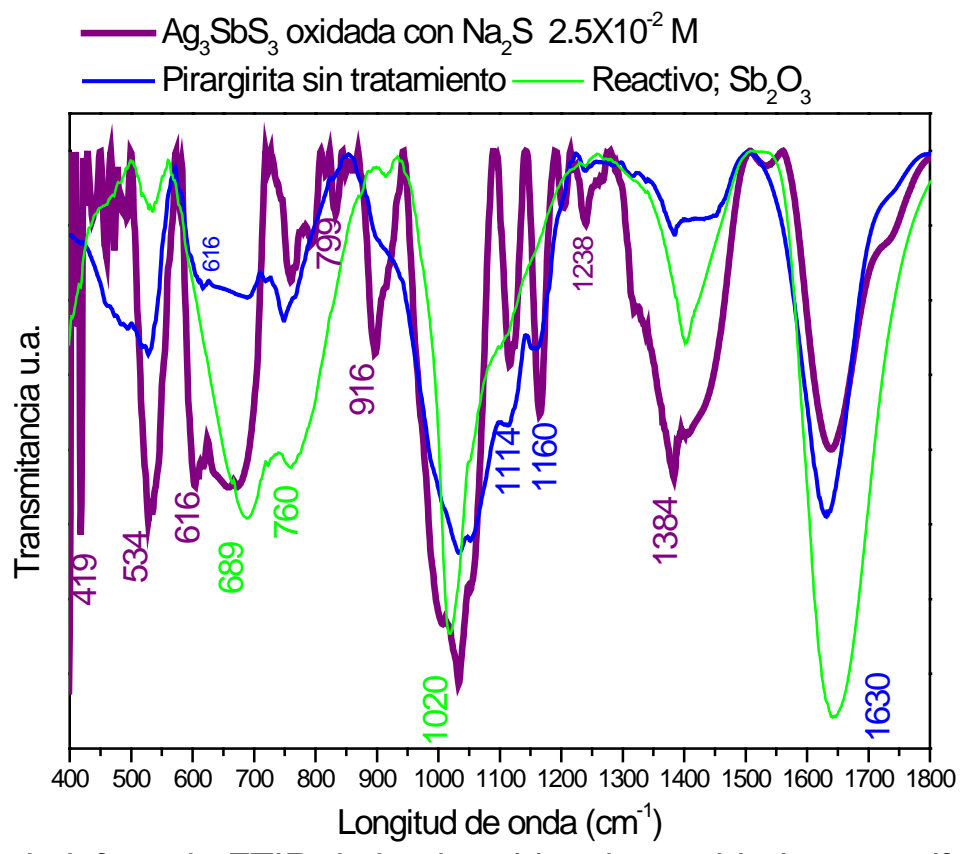

Figura 3. Espectros de infrarrojo FTIR de la pirargirita sin y oxidada con sulfuro de sodio.

Además de las bandas de absorción del trióxido de antimonio, se observan intensas bandas de absorción del ion sulfato enlazado de manera monodentada con tres bandas de absorción en $1160 \mathrm{~cm}^{-1}, 1115 \mathrm{~cm}^{-1}$ y $1033 \mathrm{~cm}^{-1}$. La banda en alrededor de $419 \mathrm{~cm}^{-1}$, indica la presencia de los enlaces $S-S$ presentes en sulfuro de plata.

\section{CONCLUSIONES}

El mineral empleado en esta investigación corresponde a una pirargirita de alta pureza con contenidos de 58.54 \% de Ag, 17.01 de S, 19.49 de Sb y Cu $4.95 \%$. El sulfuro de sodio actúa como un fuerte agente oxidante del antimonio, pasando a $\mathrm{Sb}_{2} \mathrm{~S}_{4}{ }^{2-}$ acuoso, el sólido resultante corresponde a un sulfuro de plata identificado con el patrón de difracción PDF 00 -019-1135. No obstante, la espectroscopia de infrarrojo muestra que el antimonio no se elimina en su totalidad permaneciendo superficialmente los enlaces $\mathrm{Sb}-\mathrm{O}$ del trióxido de antimonio, la presencia de esta especie resulta en la formación de capa de pasivación donde como primera aproximación se espera retarde la cianuración de la plata.

\section{AGRADECIMIENTOS}

A la Universidad Autónoma del Estado de Hidalgo, al Programa para el Desarrollo Profesional Docente PRODEP, y al Instituto Politécnico Nacional unidad ESIQUIE. 


\section{BIBLIOGRAFÍA}

[1]. M. Khalid, F. Larachi, A. Adnot. The Canadian Journal of Chemical Engineering. 95 (2017) 1875-1884.A.R. Akbashev, A.R. Kaul. Russ. Chem. Rev. 80 (2011) 11591177.

[2]. J. Zhou. Proceedings of the 42nd Annual Meeting of Canadian Mineral Processors, Ottawa, Ontario, Canada (2010) 143-161.

[3]. O. Celep, A. D. Bas, E. Y. Yazici, I. Alp, H. Deveci. Miner. Process. Extractive Metall. Rev. 36 (2015) 227-236.

[4]. C. Rodríguez Rodríguez, F. Nava-Alonso, A. Uribe-Salas, J. Viñals. Hydrometallurgy 164 (2016) 15-23.

[5]. K. S. Fraser, R. H. Walton, J. A. Wells. Processing of refractory gold ores. Miner. Eng. 4 (1991) 1029-1041.

[6]. P. Balaz. Extractive Metallurgy of Activated Minerals. Elsevier, Amsterdam. (2000) 290.

[7]. P. Balaz, J. Ficeriova, C. Villachica Leon. Hydrometallurgy. 70 (2003) 113-119 